\title{
Pathogen Distribution and Laboratory Diagnosis of Acute Respiratory Infectious Diseases
}

\author{
Zhiyong Qin', Guosheng Su${ }^{2 *}$ \\ ${ }^{1}$ Inspection Department, Guiping Municipal People’s Hospital, Guigang, China \\ ${ }^{2}$ Guangxi-ASEAN Economic Development Zone People’s Hospital/Nanning Tenth People’s Hospital Laboratory, Nanning, China \\ Email: qinzhiyong0866@163.com, ^563449581@163.com
}

How to cite this paper: Qin, Z.Y. and Su, G.S. (2021) Pathogen Distribution and Laboratory Diagnosis of Acute Respiratory Infectious Diseases. Advances in Infectious Diseases, 11, 21-28.

https://doi.org/10.4236/aid.2021.111004

Received: January 27, 2021

Accepted: March 2, 2021

Published: March 5, 2021

Copyright $\odot 2021$ by author(s) and Scientific Research Publishing Inc. This work is licensed under the Creative Commons Attribution International License (CC BY 4.0).

http://creativecommons.org/licenses/by/4.0/ (c) (i) Open Access

\begin{abstract}
Objective: To investigate the etiology distribution and laboratory diagnosis of acute respiratory infectious diseases. Methods: By searching, collecting and summarizing the etiology distribution and laboratory diagnosis of respiratory infectious diseases, the latest research progress of acute respiratory infectious diseases was studied in order to provide reference for clinical prevention and treatment. Results: In the relevant literature collected, it is considered that acute respiratory infectious diseases refer to infectious diseases caused by pathogens invading human body from the nasal cavity, throat, trachea and bronchus. The main acute respiratory infectious diseases are SARS (acute severe respiratory syndrome), MERS (Middle East respiratory syndrome), 2019-ncov infection (new coronavirus pneumonia), pulmonary plague, influenza, measles, diphtheria, pertussis, rubella, meningitis, mumps, tuberculosis, etc. Different types of infection have different diagnostic methods, and different treatments are given according to different diseases. Conclusion: The etiology distribution and laboratory diagnosis of acute respiratory tract infectious diseases are understood through reference, which can provide better reference for clinical practice. Acute respiratory infectious disease is the most common clinical disease, which seriously endangers people's health.
\end{abstract}

\section{Keywords}

Respiratory Infections, Pathogens, Clinical Diagnosis, Prevention and Control Research

\section{Introduction}

Acute Respiratory Infection (ARI) is the most common infectious disease in 
clinical diagnosis and treatment. It occurs regardless of age, gender and season. It is a disease with high morbidity and mortality among infectious diseases. According to the research of the World Health Organization, the number of deaths in the world every year is as high as 921,000 (812,000 to 1.1117 million), among which ARI causes children under 5 years old, accounting for $18.3 \%$ of the deaths in this age group [1] [2]. ARI has become the main cause of hospitalization for infants and young children and the most common reason for adults to seek medical treatment. A study from Lancet in 2018 showed that ARI is still the main acute health risk disease for adults worldwide, with approximately 291,243 - 645,832 cases deaths caused by ARI each year (4.0 8.8 cases per 100,000 people) [3]. While another study from John Paget et al. [4] 2019 indicated that in updating the global burden assessment, approximately 294,000 - 518,000 deaths per year (an average of 389,000) were found, which is equivalent to $2 \%$ of all deaths due to respiratory diseases throughout the year, $67 \%$ of which were patients over 65 years of age. COVID-2019, a new type of coronavirus pneumonia that broke out in December 2019, can be described as a global pandemic, and it has not stopped so far. As of January 12, 2021, a total of 91,229,094 confirmed cases and 1,951,071 deaths have been reported worldwide; a total of 23,090,046 confirmed cases and 384,681 deaths have been reported in the United States. Relevant studies have shown that more than $90 \%$ of ARI is infected by pathogens other than bacteria, including viruses, mycoplasma, chlamydia, rickettsia, etc., of which viruses account for about $80 \%$ [5]. Therefore, the situation of prevention and treatment of acute respiratory tract infection is severe and deserves the attention of medical workers. In order to explore the clinical diagnosis and prevention of acute respiratory infectious diseases, the author searched Wanfang database, CNKI, PubMed, MedSic and so on, and sorted out the relevant references in recent years (See the flow figure below), and summarized the results as follows (Figure 1).

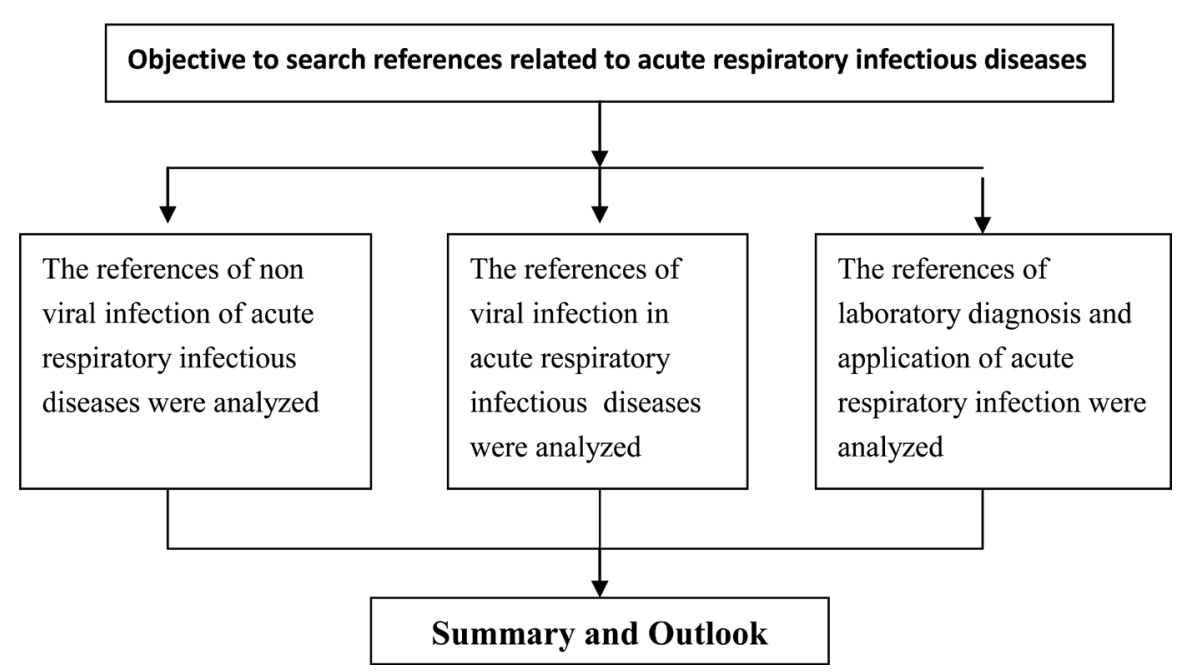

Figure 1. Summary and outlook. 


\section{Non-Viral Infections of Acute Respiratory Infectious Diseases}

The proportion of non-viral pathogens in acute respiratory infectious diseases is about $10 \%$. The common pathogens include bacteria, mycoplasma, chlamydia, Rickettsia and so on. In order to understand the etiological characteristics and diagnostic value of IgM Antibody (IgM-Ab) for Lower respiratory tract infection in children, Ren Chao et al. [6] performed indirect immunofluorescence combined detection of nine respiratory pathogen IgM antibodies from the blood samples of 1980 cases patient in children. The results found that 2 cases of Legionella pneumophila (LP) infection, accounting for $0.1 \%$; 28 cases of Mycoplasma pneumoniae (MP) infection, accounting for $1.41 \%$; 60 cases of $\mathrm{Q}$ fever Rickettsia (QFR) infection, accounting for 3.03\%; Chlamydia pneumoniae (CP) 54 cases were infected, accounting for $2.73 \%$. In order to understand the distribution and drug resistance of pathogens in COPD complicated by lower respiratory tract infections in different courses, Xi Jing et al. [7] collected 623 cases of pathogenic bacteria detected in the hospital which was divided into two groups: the course of disease group ( $>10$ years) and the short course of disease group ( $\leq 10$ years) according to the duration of COPD. As a result, there were 281 and 342 patients in the long course group and short course group, respectively; the pathogens in each course group were mainly Gram-negative bacteria. The isolation rate of gram-negative bacteria and fungi in the long course group was higher than that in the short course group $(\mathrm{P}<0.05)$. The isolation rates of Acinetobacter baumannii, Pseudomonas aeruginosa, ESBLs-producing strains and CRE strains in the long course group were higher than those in the short course group $(\mathrm{P}<0.05)$. It was considered that the pathogens of acute exacerbation of obstructive pulmonary disease (AECOPD) were mainly gram-negative bacteria, and the composition and drug resistance of pathogens were related to the course of COPD. The detection rate of multi-drug-resistant strains and fungi in the long-term group was high, and the forms of drug resistance were severe [7] [8]. Yao Yao et al. [9] analyzed the distribution and drug resistance of pathogenic bacteria of respiratory tract infections in the hospital in 2018, and the results showed that Pseudomonas aeruginosa, Klebsiella pneumoniae, Escherichia coli and Staphylococcus aureus were common bacterial respiratory infections in the elderly, and the drug resistance of various bacteria was on the rise. It is believed that the elderly patients with respiratory tract infection are mainly Gram-negative bacteria, and antibiotic resistance is on the rise. It is suggested that antibiotics should be used reasonably according to the patient's condition.

\section{Virus Infection of Acute Respiratory Infectious Diseases}

Most of the pathogens of acute respiratory infections are viral infections, so it is particularly important to study viral infections. Common viruses of respiratory tract infections are: Influenza Virus type A (INFA), Influenza Virus type B (INFB), Human Parainfluenza Virus (HPIV), Human Respiratory Syncytial Vi- 
rus (HRSV), Human Adenovirus (HADV), Human Rhinovirus (HRV), Bocavirus (Boca), Human Coronavirus (HCOV) and Human Metapneumovirus (HMPV) [10] [11]. Wang $X$ and other researchers [2] systematically evaluate 157 studies published between 1995-2018; The latest assessment of influenza virus-related respiratory tract infections in children under 5 years old found that among children under 5 years of age, about 1.095 million (0.631 - 1.906 million) cases of influenza virus infections occurred globally, of which 101 thousand (68 thousand - 1.51 million) cases had acute lower respiratory tract infections and 15 thousand (6 thousand - 44 thousand) died in hospital. The European Center for Disease Control and Prevention and WHO have reviewed the influenza situation in the entire European region from 2019 to 2020 and found that the annual influenza positive rate was $37.2 \%$, and the highest influenza positive rate was $55 \%$, which occurred in May-June. According to the latest research data in March 2020, the positive rate of influenza viruses in patients with ARI is $43 \%$, and the main influenza viruses are influenza A and B (59\% and 41\%). Influenza A viruses are composed of H1N1 (2009) (56\%) and H3N2 (44\%), the majority of influenza B viruses are of the B/Victoria lineage (99\%) [12]. At the same time, the fourth meeting of the Eastern Mediterranean ARI Surveillance Network pointed out that a large part of the global ARI burden of children and the elderly is attributed to influenza virus and respiratory syncytial virus [13]. In addition, 30\% of adult patients with Community Acquired Pneumonia (CAP) are caused by respiratory virus infection, and the mortality rate is as high as $3 \%$ [14]. Due to a series of factors such as hunting and eating wild animals, climate change, melting glaciers, injecting drugs, poor personal hygiene habits, etc., not only emerging pathogenic bacteria can be produced, but also the evolution or mutation of pathogens can be caused to obtain antibiotic resistance or enhance virulence, and even evolved into unknown human pathogens, such as SARS-Cov [15] [16].

\section{Laboratory Diagnosis and Application of Acute Respiratory Infections}

At present, the laboratory diagnosis of acute respiratory tract infection is changing with each passing day. Because the pathogens of respiratory tract infection are complex and changeable, pathogen detection has become the biggest challenge for laboratory diagnosis. Bacterial respiratory tract infection can be isolated and identified by sputum bacterial culture to obtain accurate pathogen and drug sensitivity analysis. And the manifestations caused by different respiratory non-bacterial pathogens can range from no obvious symptoms to serious illness. Doctors may not be able to make an accurate diagnosis of the cause based on clinical manifestations alone. How to efficiently, quickly and accurately detect the pathogens infected by patients is a clinical difficulty [17] [18]. In recent years, the detection methods of non-bacterial pathogens are changing day by day: including fractional cultivation, serum antibody method, and molecular biology techniques for nucleic acid detection. Nucleic acid amplification tech- 
niques represented by conventional polymerase chain reaction (PCR), reverse transcription polymerase chain reaction (RT-PCR) and multiplex PCR have been widely used in clinical practice [18] [19] [20]. Molecular biology technology detection has the characteristics of high efficiency and speed, which is not only conducive to early acquisition of pathogen information of ARI patients, but also conducive to the rational selection of effective drugs, and the rapid recovery of patients. Capillary electrophoresis fragment analysis technology, namely Capillary Electrophoresis (CE), is a new and efficient analysis and separation technology developed from traditional electrophoresis. It was first proposed by Jorgenson and Lukace in 1981 [21]. CE has been widely used in nucleic acid separation analysis, protein, amino acid, drug analysis, etc., with high sensitivity and high separation selectivity [22] [23]. Because CE technology has both electrophoresis and chromatography functions, the separation efficiency of pathogens is higher. When CE and multiple T-PCR technology are used in combination, the sensitivity and specificity of detection can be improved. Stevenson JB et al. [24] used this technique to detect the three pathogens of HRSV, InfA and InfB in 30 positive samples and found that 2 samples could not be detected due to low content. The overall result showed that the detection rate reached $93 \%$. At the same time, Jiang LX et al. [25] used multiplex RT-PCR combined with capillary electrophoresis to detect 13 ALRI bacterial pathogens in 152 samples, and the results showed a specificity of $96.5 \%-100 \%$ and a sensitivity of $100 \%$ which further shows that multiple RT-PCR combined with capillary electrophoresis is a fast, reliable, and high-throughput method with high sensitivity and specificity. In summary, the monitoring of the etiology of ARI is not only conducive to the prediction, prevention and control of the ARI epidemic, but also provides a theoretical basis for the diagnosis and treatment of ARI, and also reduces the use of unnecessary antibiotics.

\section{Summary and Outlook}

Through the reference of the literature, we understand the pathogenic distribution of the current acute respiratory infectious diseases and the laboratory diagnosis plan, which can provide a better reference for the clinic. ARI is the most common infectious disease in human beings. Most pathogenic microorganisms (such as viruses, bacteria, fungi, parasites, etc.) can cause ARI, among which viruses are the main pathogen, and the clinical symptoms can range from no obvious symptoms to serious diseases. Since most of the symptoms after infection with non-bacterial pathogens are similar, it is difficult for doctors to make an accurate diagnosis based on the patient's symptoms, let alone identify the type of pathogen.

Traditional methods for identifying respiratory pathogens include sputum Gram staining, respiratory secretion culture and blood serological examination [26]. Although these traditional methods can identify respiratory pathogens to some extent, their disadvantages cannot be ignored, such as time-consuming, 
labor-intensive and poor sensitivity. In order to detect quickly and identify respiratory pathogens, the technologies represented by molecular detection methods such as nucleic acid PCR are emerging, which have high sensitivity and specificity, and can be used to analyze clinical samples such as blood, sputum and urine, and improve the diagnostic ability of patients with respiratory disease [27] [28]. The application of multiplex RT-PCR technology can detect many pathogens at one time in the experiment, which greatly shortens the detection time and is more reliable and convenient than virus culture. Several studies have reported successful detection of respiratory pathogens by multiplex RT-PCR [29] [30]. At the same time, the emerging gene sequencing technology is producing more accurate and rapid results for identifying these pathogens. Viruses have become the most common pathogenic non-bacterial pathogen in ARI patients, because they have similar clinical manifestations and may appear in various forms of infection, which further complicates ARI and increases the incidence. Conventional detection methods are laborious and time-consuming, while multiplex RT-PCR combined with capillary electrophoresis analysis is fast, labor-saving and high-throughput, which is very important for enhancing the detection and identification of respiratory pathogens in ARI clinics. Acute respiratory infectious diseases are the most common diseases in clinic, which seriously endanger people's health. It is necessary to see a doctor in time so as to get diagnosis and treatment as soon as possible.

\section{Acknowledgements}

During the process of this topic research, we got much help from many departments and individuals, and other personnel not involved in this project research. All of them offered great support and help in this research. Now here, all of members in this research show our deepest appreciation to them, and wish them good health and everything goes well.

\section{Funding}

Guigang scientific research and technology development project (NO.: Guigang science and technology 1803028).

\section{Conflicts of Interest}

The authors declare no conflicts of interest regarding the publication of this paper.

\section{References}

[1] Liu, L., Oza, S., Hogan, D., et al. (2016) Global, Regional, and National Causes of Under-5 Mortality in 2000-15: An Updated Systematic Analysis with Implications for the Sustainable Development Goals. The Lancet, 388, 3027-3035. https://doi.org/10.1016/S0140-6736(16)31593-8

[2] Wang, X., Li, Y., O’Brien, K.L., et al. (2020) Global Burden of Respiratory Infections 
Associated with Seasonal Influenza in Children under 5 Years in 2018: A Systematic Review and Modelling Study. The Lancet Global Health, 8, e497-e510. https://doi.org/10.1016/S2214-109X(19)30545-5

[3] Iuliano, A.D., Roguski, K.M., Chang, H.H., et al. (2018) Estimates of Global Seasonal Influenza-Associated Respiratory Mortality: A Modelling Study. The Lancet, 391, 1285-1300. https://doi.org/10.1016/S0140-6736(17)33293-2

[4] Paget, J., Spreeuwenberg, P., Charu, V., et al. (2019) Global Mortality Associated with Seasonal Influenza Epidemics: New Burden Estimates and Predictors from the GLaMOR Project. Journal of Global Health, 9, Article ID: 020421. https://doi.org/10.7189/jogh.09.020421

[5] Richter, J., Panayiotou, C., Tryfonos, C., et al. (2016) Aetiology of Acute Respiratory Tract Infections in Hospitalised Children in Cyprus. PLoS ONE, 11, e147041. https://doi.org/10.1371/journal.pone.0147041

[6] Ren, C. (2020) Study on Pathogens and Infection Indexes of Children's Lower Respiratory Tract Infection. Nanchang University, Nanchang.

[7] Xi, J., Tuo, M.F., Wei, Y.F., Dai, H.Y., Qiu, H.L. and Li, H.K. (2020) Isolation and Drug Resistance Analysis of Pathogenic Bacteria of COPD Complicated with Lower Respiratory Tract Infection in Different Courses. Chinese Journal of Antibiotics, 45, 798-803.

[8] Liu, Y.Y., Yang, J.H. and Ye, D. (2019) Analysis of Risk Factors of Respiratory Multi-Drug Resistant Bacteria Infection in Patients with Acute Exacerbation of Chronic Obstructive Pulmonary Disease. Journal of Clinical Pulmonary Medicine, 24, 661-664.

[9] Yao, Y., Gao, H., Huang, Y.K., Zhu, W.M. and Wang, J. (2020) A Third Class Hospital in Kunming; Distribution and Drug Resistance Analysis of Pathogenic Bacteria of Respiratory Tract Infection in Geriatrics Department in Kunming in 2018. Chinese Journal of Antibiotics, 45, 166-169.

[10] Al-Tawfiq, J.A., Zumla, A., Gautret, P., et al. (2014) Surveillance for Emerging Respiratory Viruses. The Lancet Infectious Diseases, 14, 992-1000. https://doi.org/10.1016/S1473-3099(14)70840-0

[11] Dunn, J.J. and Miller, M.B. (2014) Emerging Respiratory Viruses Other than Influenza. Clinics in Laboratory Medicine, 34, 409-430. https://doi.org/10.1016/j.cll.2014.02.011

[12] European Centre for Disease Prevention and Control/WHO Regional Office for Europe (2020) Flu News Europe, Joint ECDC-WHO Weekly Influenza Update, Week 10/2020.

[13] Abubakar, A., Barakat, A., Ahmed, A., et al. (2020) Fourth Meeting of the Eastern Mediterranean Acute Respiratory Infection Surveillance (EMARIS) Network and First Scientific Conference on Acute Respiratory Infections in the Eastern Mediterranean Region, 11-14 December, 2017, Amman, Jordan. Journal of Infection and Public Health, 13, 451-456. https://doi.org/10.1016/j.jiph.2020.02.031

[14] Ishiguro, T., Takayanagi, N., Yamaguchi, S., et al. (2013) Etiology and Factors Contributing to the Severity and Mortality of Community-Acquired Pneumonia. Internal Medicine, 52, 317-324. https://doi.org/10.2169/internalmedicine.52.8830

[15] Menachery, V.D., Schafer, A., Burnum-Johnson, K.E., et al. (2018) MERS-CoV and H5N1 Influenza Virus Antagonize Antigen Presentation by Altering the Epigenetic Landscape. Proceedings of the National Academy of Sciences of the United States of America, 115, E1012-E1021. https://doi.org/10.1073/pnas.1706928115

[16] Otter, J.A., Donskey, C., Yezli, S., et al. (2016) Transmission of SARS and MERS 
Coronaviruses and Influenza Virus in Healthcare Settings: The Possible Role of Dry Surface Contamination. Journal of Hospital Infection, 92, 235-250.

https://doi.org/10.1016/j.jhin.2015.08.027

[17] Lin, C.Y., Hwang, D., Chiu, N.C., et al. (2020) Increased Detection of Viruses in Children with Respiratory Tract Infection Using PCR. International Journal of Environmental Research and Public Health, 17, 564.

https://doi.org/10.3390/ijerph17020564

[18] Zhang, N., Wang, L., Deng, X., et al. (2020) Recent Advances in the Detection of Respiratory Virus Infection in Humans. Journal of Medical Virology, 92, 408-417. https://doi.org/10.1002/jmv.25674

[19] Zhao, L., Li, G.X., Wang, J., et al. (2020) Development and Evaluation of a Panel of Multiplex One-Tube Nested Real Time PCR Assay for Simultaneous Detection of 14 Respiratory Viruses in Five Reactions. Journal of Medical Virology. https://doi.org/10.1002/jmv.25686

[20] Peng, X. (2020) Distribution of Nonbacterial Pathogens in Outpatients with Acute Respiratory Infections. Nanchang University, Nanchang.

[21] Jorgenson, J.W. and Lukacs, K.D. (1981) Free-Zone Electrophoresis in Glass Capillaries. Clinical Chemistry, 27, 1551-1553. https://doi.org/10.1093/clinchem/27.9.1551

[22] Landers, J.P., Oda, R.P., Spelsberg, T.C., et al. (1993) Capillary Electrophoresis: A Powerful Microanalytical Technique for Biologically Active Molecules. Biotechniques, 14, 98-111.

[23] Grabarics, M., Csernak, O., Balogh, R., et al. (2017) Analytical Characterization of Human Milk Oligosaccharides-Potential Applications in Pharmaceutical Analysis. Journal of Pharmaceutical and Biomedical Analysis, 146, 168-178. https://doi.org/10.1016/j.jpba.2017.08.039

[24] Stevenson, J.B., Hymas, W.C. and Hillyard, D.R. (2011) A Novel Capillary Electrophoresis-Based Multiplex PCR Assay for Detection of Respiratory Pathogens. Annals of Clinical \& Laboratory Science, 41, 33-38.

[25] Jiang, L.X., Ren, H.Y., Zhou, H.J., et al. (2017) Simultaneous Detection of 13 Key Bacterial Respiratory Pathogens by Combination of Multiplex PCR and Capillary Electrophoresis. Biomedical and Environmental Sciences, 30, 549-561.

[26] Mandell, L.A. (2015) Community-Acquired Pneumonia: An Overview. Postgraduate Medicine, 127, 607-615. https://doi.org/10.1080/00325481.2015.1074030

[27] Pai, R., Gertz, R.E. and Beall, B. (2006) Sequential Multiplex PCR Approach for Determining Capsular Serotypes of Streptococcus pneumoniae Isolates. Journal of Clinical Microbiology, 44, 124-131. https://doi.org/10.1128/JCM.44.1.124-131.2006

[28] Tian, G.Z., Zhang, L.J., Wang, X.L., et al. (2012) Rapid Detection of Haemophilus influenzae and Haemophilus parainfluenzae in Nasopharyngeal Swabs by Multiplex PCR. Biomedical and Environmental Sciences, 25, 367-371.

[29] Li, J., Mao, N.Y., Zhang, C., et al. (2012) The Development of a GeXP-Based Multiplex Reverse Transcription-PCR Assay for Simultaneous Detection of Sixteen Human Respiratory Virus Types/Subtypes. BMC Infectious Diseases, 12, 189. https://doi.org/10.1186/1471-2334-12-189

[30] Wang, L., Zhao, M., Shi, Z., et al. (2016) A GeXP-Based Assay for Simultaneous Detection of Multiple Viruses in Hospitalized Children with Community Acquired Pneumonia. PLoS ONE, 11, e162411. https://doi.org/10.1371/journal.pone.0162411 